\author{
ARTIFICIAL SATELLITES, Vol. 42, No. 2 - 2007 \\ DOI: 10.2478/v10018-008-0004-1
}

\title{
DETERMINATION OF THE GOCE SATELLITE ORBIT SENSITIVITY UNDER THE INFLUENCE OF PERTURBING FORCES
}

\author{
A. Bobojć, A. Drożyner \\ University of Warmia and Mazury in Olsztyn, Institute of Geodesy, Olsztyn, Poland \\ e-mail: altair@uwm.edu.pl,drozyner@uwm.edu.pl
}

\begin{abstract}
The work contains the results of research into the simulated orbit of the GOCE satellite. Satellite accelerations due to atmospheric drag, the gravitation of the Moon, the gravitation of the Sun, the gravitation of the planets, the Earth tides, the ocean tides, the solar radiation pressure, the reflected solar radiation pressure and the relativity effects were computed. Besides the reference orbit (i.e. the orbit as close as possible to the GOCE planned orbit), the various variants of the satellite orbit (called the modified reference orbits - with different motion models) were obtained. The motion models contained the forces determining the satellite motion. For the orbital computations, the Cowell numerical integration of the eighth order was used. The geopotential was described by means of the EGM96 model.

In order to obtain the influence of the aforementioned forces on the GOCE orbit, the percentage contributions of the accelerations due to these forces in the sum of all accelerations were computed.

The maximum values of the mentioned accelerations were computed for the GOCE orbit variant with the motion model containing all given above forces. These values were compared with the measurement error of the linear accelerations by the GOCE satellite control system.

The comparison between the reference orbit and the computed variants of the orbit was performed. In order to perform this comparison, the distances between the satellite position in the reference orbit and the satellite position in the given modified reference orbit (i.e. in a given orbit variant) were determined. These distances were compared with the total error of the GOCE satellite position determination. The orbital arc lengths, for which the selected forces can be neglected in the satellite motion model, were determined from this comparison. For these orbital arc lengths, the distance between the satellite position in the reference orbit and the satellite position in the given modified reference orbit is less or equal to the total error of the GOCE satellite position determination.
\end{abstract}

Key words: satellite orbit, GOCE mission, perturbing forces.

\section{INTRODUCTION}

The Gravity Field and Steady - State Ocean Circulation Explorer Mission (GOCE) is being developed as part of the Living Planet Programme of ESA. The GOCE mission will provide an unprecedented model of the Earth's gravity field. The derived model will make it possible to estimate the gravity acceleration and the geoid with an accuracy of $1 \mathrm{mGal}\left(10^{-5} \mathrm{~m} / \mathrm{s}^{2}\right)$ and 
of $1 \mathrm{~cm}$, respectively, with a resolution of $100 \mathrm{~km}$ (Drinkwater et al., 2003; Mégie and Readings, 2000, Rebhan et al., 2000). A 20-month lifetime with two 6-month observation periods is planned for the mission (ESA, 1999; ESA, 2006).

The GOCE satellite is planned for launch from Russia in 2008 and is intended to be placed in a near circular orbit with an average altitude of about $250 \mathrm{~km}$ (ESA, 2006). The inclination of the orbit is planned to be 96.5 degrees, which corresponds to the sun-synchronous orbit (ESA, 1999). The main sensor of the GOCE satellite will be an electrostatic gravity gradiometer. An on-board GPS/GLONASS receiver and a drag-free control system for nongravitational forces compensation will be the important elements of the satellite (Wermuth et al.,2006).

The GOCE mission is designed to use two observation techniques: Satellite Gravity Gradiometry (SGG) and High-Low Satellite to Satellite Tracking (SST). The SGG data (i.e. gravity gradients - the second spatial derivatives of the geopotential) will be provided by the gradiometer, whereas the SST data (GPS/GLONASS) will be obtained using the GPS/GLONASS receiver (Johannessen et al., 2003). Both data types are complementary. The SST technique is more sensitive in the long periods, whereas the SGG is more sensitive to the short wavelength components of the gravity field of the Earth. Six 3-axes accelerometers mounted in pairs along three orthogonal arms constitute the core of gradiometer. The accelerations measured by accelerometers will provide two types of data. Differential mode measurements will be used to obtain the gravity gradients, whereas common mode observations will provide data on the linear accelerations for the drag-free control system. The precision of the gravity gradient determination will not exceed $4 \mathrm{mE} / \sqrt{\mathrm{Hz}}\left(1 \mathrm{mE}=10^{-12} \mathrm{~s}^{-2}\right)$ in the measurement bandwidth of the gradiometer between 5 and $100 \mathrm{mHz}$ (Bouman and Koop, 2003).

Advanced algorithms of the determination of the Earth's gravity field model using simulated GOCE measurements have already been developed. These algorithms indicate that the joint inversion of both SGG and SST data provides a more accurate gravity field model than a stand-alone inversion of SST or SGG data (Ditmar and Klees, 2002; Ditmar et al., 2003).

A very important factor in the process of gravity field model estimation is knowledge of the GOCE satellite orbit. GPS measurements from the onboard GPS receiver will be the basic observations for the GOCE satellite orbit determination. The European GOCE GravityConsortium has created the GOCE High-level Processing Facility dedicated to data processing. This facility includes, among others, the computation of the Precise Science Orbit for GOCE as a reduced-dynamic and a kinematic orbit solution (Bock et al., 2006; Visser at al.,2006).

In addition to the GPS observations, the SGG measurements from the GOCE gradiometer also contain data on the position and velocity of the satellite. Simulations indicating the possibility of improving satellite orbit by using gravity gradients were performed (Bobojć and Drożyner, 2003). However, the orbit improvement process requires the computation of approximated observations (i.e. gravity gradients) along an approximated satellite orbit. The objective of this work was to obtain the approximated GOCE satellite orbits which could be used in the orbit improvement process. These orbits were generated by using different models of satellite motion. The satellite motion models contained different sets of the forces determining the satellite motion. The values of satellite accelerations generated by perturbing forces for the GOCE orbit were estimated in order to determine the influence of these forces on this orbit. The computed accelerations will be applied in the planned simulation of the GOCE gradiometer measurements. 


\section{SIMULATIONS}

In this work, the GOCE satellite orbit was computed with respect to the J2000.0 reference frame (Laing, 1991, Anderson et al., 2002). This frame is defined in the following manner: the origin at the Earth's mass centre, the $\mathrm{X}$-axis is directed towards the mean vernal equinox of the standard epoch J2000.0 (at noon on January 1, 2000), the Z-axis points out from the Earth's mass centre along the Earth's mean rotational axis of the standard epoch J2000.0, the $\mathrm{Y}$-axis completes the frame to the right-handed frame. The following initial elements of the GOCE satellite orbit were included in the computation: the epoch: 54313.0 MJD (2007-08-01 $00 \mathrm{~h} 00 \mathrm{~m} 00 \mathrm{~s} \mathrm{UTC}$ ), semi-major axis: $6634.7711 \mathrm{~km}$, eccentricity: 0.001 , inclination: 96.5 deg., argument of perigee: $0.00 \mathrm{deg}$., right ascension of ascending node: $45.00 \mathrm{deg}$, mean anomaly: $0.00 \mathrm{deg}$., the orbital period $\mathrm{T}=89.64 \mathrm{~min}$. and the altitude: $250.00 \mathrm{~km}$ (ESA,1999). All computations were performed employing the TOP package (Drożyner, 1995). This package generates a satellite orbit in the Earth's gravity field taking into account the aforementioned perturbing forces. The Cowell numerical integration method of the eighth order is implemented in this package.

Several force models were used. For the geopotential, the EGM96 model was taken (Lemoine et al.,1998). The following models were taken from the TOP package: MERIT Standards - the Earth and ocean tides (Melbourne et al. 1983), CIRA-72 - the atmosphere (CIRA-72, 1972). The models describing the relativity effects and direct and reflected solar radiation pressure also originated from this package. The computations were performed taking into account the Sun, Moon and planetary ephemerides DE200/LE200 (epoch J2000.0), the geomagnetic indices (from the National Geophysical Data Center USA for 1996 - the extrapolation to 2007 took into account the Sun's 11-year cycle), the IAU1976 Theory of Precession and the IAU1980 Theory of Nutation (the Wahr nutation). The pole coordinates were neglected due to the difficulties in prediction.

A 30-day arc of the reference orbit, i.e. an orbit as close as possible to the planned GOCE satellite orbit, was simulated. The geopotential and the selected perturbing forces determined the satellite motion in this orbit. The selected perturbing forces included the gravitation of the Moon, the gravitation of the Sun, the gravitation of the planets, the Earth and ocean tides and the relativity effects.

The non-gravitational forces will be compensated by a special control system in the GOCE satellite (Drinkwater et al., 2003). However, the 30-day arc of the complete orbit, i.e. the orbit based on the planned initial elements of the GOCE satellite orbit was also computed, but without compensation for the non-gravitational forces. This orbit was determined by the same forces as for the reference orbit and additionally by the nongravitational forces. These forces included: the atmospheric drag, the direct solar radiation pressure and the reflected solar radiation pressure. Both the reference orbit and the complete orbit were obtained using a $60 \mathrm{~s}$ integration step. Additionally, the various variants of the GOCE satellite orbit were determined. The satellite motion models for these orbital variants were obtained by subtracting or adding the selected forces to the satellite motion model for the reference orbit. Thus, the obtained orbit variants can be called the modified reference orbits.

To compare the modelled forces acting on the satellite, the corresponding accelerations were computed along the complete orbit and the reference orbit. In addition, the various arc lengths of the modified reference orbits were obtained. For these arc lengths, the distance between the satellite position in the reference orbit and the satellite position in the given modified reference orbit reaches the value of the expected total error of the GOCE position determination. 


\section{RESULTS}

In order to compare the given forces acting on the satellite, the maximum accelerations due to these forces for the complete orbit were shown in the second column of Table 1.

Table 1. The maximum values of accelerations for the complete orbit (the orbital arc length -30 days, the initial epoch - 54313.0 MJD, the integration step - $60 \mathrm{~s}$ ) (second column). The contribution of the acceleration for the reference orbit due to the given force in the sum of all accelerations (fourth column). The presented contributions are the averages from the analogous contributions in all epochs of the reference orbit (with the non-gravitational forces compensation, the orbital arc length - 30 days). Above the bold line - the contribution of the acceleration due to the geopotential and the contribution of the acceleration due to the perturbing forces. Below the bold line - the acceleration contributions for the perturbing forces (without the geopotential).

\begin{tabular}{|c|c|c|c|}
\hline FORCE & $\begin{array}{c}\text { ACCELERATION } \\
{\left[\mathrm{m} / \mathrm{s}^{2}\right]}\end{array}$ & FORCE & $\begin{array}{c}\text { CONTRIBUTION } \\
{[\%]}\end{array}$ \\
\hline Geopotential & 9.339942 & Geopotential & 99.99998 \\
\hline Atmospheric drag & $1.115690 \cdot 10^{-4} \quad$ (c) & Perturbing forces & 0.00002 \\
\hline Gravitation of the Moon & $1.310619 \cdot 10^{-6}$ & Gravitation of the Moon & 54.60826 \\
\hline Ocean tides & $4.060530 \cdot 10^{-7}$ & Gravitation of the Sun & 19.35963 \\
\hline Earth tides & $4.017976 \cdot 10^{-7}$ & Earth tides & 19.27024 \\
\hline Gravitation of the Sun & $2.680858 \cdot 10^{-7}$ & Ocean tides & 5.39192 \\
\hline Solar radiation pressure & $8.820186 \cdot 10^{-8} \quad$ (c) & Relativity effects & 1.36782 \\
\hline Relativity effects & $1.901639 \cdot 10^{-8} \quad(b)$ & Gravitation of Venus & $1.88693 \cdot 10^{-3}$ \\
\hline Reflected solar radiation pres. & $4.576330 \cdot 10^{-9} \quad(b)$ & Gravitation of Jupiter & $2.38032 \cdot 10^{-4}$ \\
\hline Gravitation of Venus & $2.843683 \cdot 10^{-11(b)}$ & Gravitation of Saturn & $5.38759 \cdot 10^{-6}$ \\
\hline Gravitation of Jupiter & $4.529790 \cdot 10^{-12(b)}$ & Gravitation of Mars & $5.09653 \cdot 10^{-6}$ \\
\hline Gravitation of Mars & $1.085592 \cdot 10^{-13(b)}$ & Gravitation of Mercury & $1.52015 \cdot 10^{-6}$ \\
\hline Gravitation of Saturn & $7.807084 \cdot 10^{-14(b)}$ & Gravitation of Uranus & $1.31842 \cdot 10^{-7}$ \\
\hline Gravitation of Mercury & $3.656653 \cdot 10^{-14(b)}$ & Gravitation of Neptune & $4.31302 \cdot 10^{-8}$ \\
\hline Gravitation of Uranus & $2.225687 \cdot 10^{-15(b)}$ & Gravitation of Pluto & $7.13416 \cdot 10^{-12}$ \\
\hline Gravitation of Neptune & $6.632677 \cdot 10^{-16(b)}$ & \multirow{2}{*}{\multicolumn{2}{|c|}{$\begin{array}{l}\text { (b) accelerations below the error of LAM * } \\
\text { (c) accelerations compensated by SCS } * *\end{array}$}} \\
\hline Gravitation of Pluto & $1.270627 \cdot 10^{-19(b)}$ & & \\
\hline
\end{tabular}

* the error of LAM - error of the linear acceleration measurement by the GOCE satellite control system $\left(4.3 \cdot 10^{-8} \mathrm{~m} / \mathrm{s}^{2},(\mathrm{ESA}, 1999)\right), * * \mathrm{SCS}-\mathrm{GOCE}$ satellite control system 
The perturbing accelerations occur in the range between $10^{-19} \mathrm{~m} / \mathrm{s}^{2}$ (generated by the gravitation of Pluto) to $10^{-4} \mathrm{~m} / \mathrm{s}^{2}$ (generated by the atmospheric drag). As mentioned, the non-gravitational forces, i.e. the atmospheric drag, the solar radiation pressure and the reflected solar radiation pressure, will be compensated. However, Table 1 shows that the maximum acceleration caused by the reflected solar radiation pressure is less than the error of the linear acceleration measurement by the GOCE satellite control system $\left(4.3 \cdot 10^{-8} \mathrm{~m} / \mathrm{s}^{2}\right.$ (ESA,1999)). Similar values of the maximum accelerations can be noticed for the ocean tides and the Earth tides, approx. $4.06 \cdot 10^{-7} \mathrm{~m} / \mathrm{s}^{2}$ and $4.02 \cdot 10^{-7} \mathrm{~m} / \mathrm{s}^{2}$, respectively.

The fourth column of Table 1 contains the percentage contributions of the accelerations for the reference orbit due to the modelled forces in the sum of all modelled accelerations for this orbit. The presented contributions were computed as the averages from the analogous contributions in all the epochs of the reference orbit (with the non-gravitational forces compensation, the orbital arc length - 30 days). In the group of perturbing forces (without the geopotential), the gravitation of the Moon, the gravitation of the Sun and the Earth tides (the contributions are approx. 54.6\%, 19.4\% and $19.3 \%$, respectively) have the largest impact on the satellite motion. The ocean tides and the relativity effects give smaller contributions of about $5.4 \%$ and $1.4 \%$, respectively. Finally, the contributions of the gravitation of the planets are smaller than $2 \cdot 10^{-3} \%$.

The arc lengths of the modified reference orbits, i.e. the approximated GOCE orbits obtained by subtracting or adding the selected forces to the satellite motion model of the reference orbit, are given in Table 2. The distances between the satellite position in the reference orbit and the satellite position in the modified reference orbits were compared with the expected total error of the GOCE position determination - about $2.8 \mathrm{~cm}$ (ESA, 1999). From this comparison, the various arc lengths of the modified reference orbits were obtained. For such orbital arc lengths, the distance between the satellite position in the reference orbit and the satellite position in the given modified reference orbit is less or equal to the aforementioned error of the GOCE position estimation. Thus, taking into account the value of this error, the forces generating the difference between the reference orbit and the given modified reference orbit can be neglected in the computation of GOCE orbit for the orbital arc lengths equal or less than the ones shown in Table 2. The values of the obtained arc lengths also depend on the forces modifying the satellite motion model of the reference orbit. The smaller the orbital arc, the greater the impact of the given force or forces on the satellite motion. The smallest orbital arcs of 1.0 minute can be noticed for the modified reference orbits with the satellite motion models obtained by adding the atmospheric drag or the nongravitational forces (with the atmospheric drag) to the motion model of the reference orbit. From among the perturbing forces presented in Table 2, the atmospheric drag has the largest impact on the satellite motion. The orbital arc with the satellite motion model, determined by subtracting the gravitation of the Moon from the motion model of the reference orbit, has a length of 4.6 minutes. However, the orbital arc with the satellite motion model obtained by subtracting the force group with the gravitation of the Moon, the gravitation of the Sun and the gravitation of the planets from the motion model of the reference orbit, has a slightly longer length of 5.0 minutes. Similarly, the orbital arc with the satellite motion model determined by subtracting the Earth tides from the motion model of the reference orbit, is 7.2 minutes long, whereas the orbital arc with the satellite motion model obtained by subtracting the tide group, i.e. the Earth tides and the ocean tides, has a length of 7.3 minutes. The Sun gravitational impact (length of the orbital arc of 7.7 minutes) is slightly smaller than the Earth tide impact (length of the orbital arc of 7.2 minutes). Next, the solar radiation pressure impact on the satellite motion is expressed by the orbital arc of 13.8 minutes. Similar values of the arc lengths can be noticed for the relativity effects and the ocean tides -25.9 minutes 
and 27.2 minutes, respectively. The length of orbital arc with the satellite motion model obtained by adding the reflected solar radiation pressure to the motion model of the reference orbit, significantly differs from previously-mentioned values and equals 128.3 minutes. The planet impacts on the satellite motion are expressed by the orbital arc lengths from 8.0 days (for Venus - the largest impact) to over 30 days (for the remaining planets). For such long orbital arcs, the gravitation of the planets can be neglected in the satellite motion model, taking into account the expected error of the GOCE satellite position determination.

Table 2. The orbital arc lengths for which the distance between the satellite position in the reference orbit and the satellite position in the given modified reference orbit is less or equal to the total error of the GOCE satellite position determination (about $3 \mathrm{~cm}$ ). The modified reference orbits were obtained by the modification of the satellite motion model for the reference orbit. For example, the "+ Ngr" notation means the non-gravitational forces adding to the satellite motion model of the reference orbit.

\begin{tabular}{|c|c|c|c|}
\hline $\begin{array}{l}\text { Reference orbit } \\
\text { modification }\end{array}$ & $\begin{array}{l}\text { Orbital } \\
\text { arc length }\end{array}$ & \begin{tabular}{|c|}
$\begin{array}{c}\text { Reference orbit } \\
\text { modification }\end{array}$ \\
\end{tabular} & $\begin{array}{l}\text { Orbital } \\
\text { arc length }\end{array}$ \\
\hline+ Atmospheric drag & $1.0 \mathrm{~min}$. & $+\mathrm{Ngr}$ & $1.0 \mathrm{~min}$. \\
\hline - Gravitation of the Moon & $4.6 \mathrm{~min}$. & $-\mathrm{MSpl}$ & $5.0 \mathrm{~min}$. \\
\hline - Earth tides & $7.2 \mathrm{~min}$. & - Tds & $7.3 \mathrm{~min}$. \\
\hline - Gravitation of the Sun & $7.7 \mathrm{~min}$. & $-\mathrm{Pl}$ & 7.8 days \\
\hline+ Solar radiation pressure & $13.8 \mathrm{~min}$. & \multirow{2}{*}{\multicolumn{2}{|c|}{$\begin{array}{l}\text { Ngr - the non- } \\
\text { gravitational forces } \\
\mathrm{MSpl} \text { - the Moon, the Sun } \\
\text { and the planets }\end{array}$}} \\
\hline - Relativity effects & $25.9 \mathrm{~min}$. & & \\
\hline - Ocean tides & $27.2 \min$. & \multirow{3}{*}{\multicolumn{2}{|c|}{$\begin{array}{l}\text { Tds - the Earth tides } \\
\text { and the ocean tides } \\
\mathrm{Pl} \text { - the planets }\end{array}$}} \\
\hline $\begin{array}{l}+ \text { Reflected solar } \\
\text { radiation pressure }\end{array}$ & $128.3 \mathrm{~min}$. & & \\
\hline - Gravitation of Venus & 8.0 days & & \\
\hline - Gravitation of Jupiter * & $>30$ days & \multirow{7}{*}{\multicolumn{2}{|c|}{$\begin{array}{l}\text { * The sequence from } \\
\text { the gravitation of Jupiter } \\
\text { to the gravitation of Pluto } \\
\text { according to the decreasing } \\
\text { distance between the satellite } \\
\text { position in the reference orbit } \\
\text { and the satellite position in } \\
\text { the given modified reference } \\
\text { orbit for the last epochs of the } \\
30 \text { - day arcs of these orbits. } \\
\text { The smaller distance for the } \\
\text { last epochs of arcs, the } \\
\text { smaller the impact of the } \\
\text { gravitation of planet on the } \\
\text { satellite motion }\end{array}$}} \\
\hline - Gravitation of Saturn & $>30$ days & & \\
\hline - Gravitation of Mars & $>30$ days & & \\
\hline - Gravitation of Mercury & $>30$ days & & \\
\hline - Gravitation of Uranus & $>30$ days & & \\
\hline - Gravitation of Neptune & $>30$ days & & \\
\hline - Gravitation of Pluto & $>30$ days & & \\
\hline
\end{tabular}




\section{SUMMARY}

The expected values of the accelerations generated by selected forces along the GOCE orbit were compared. Taking into account the perturbing forces; the gravitation of the Moon, the gravitation of the Sun and the tides (with the assumption of compensation for nongravitational forces) are the largest contributors to GOCE satellite motion. The impact of selected forces on the GOCE orbit was also expressed by the obtained orbital arc lengths from 1 minute to 8 days. These approximated GOCE satellite orbits were obtained by subtracting or adding the selected perturbing forces to the satellite motion model of the reference orbit, i.e. the orbit close to the planned GOCE orbit. The arc lengths of these orbits are relevant to the distance between the GOCE satellite position in the given approximated orbit and the GOCE satellite position in the reference orbit close to the planned orbit of this satellite. In the last epoch of the given approximated orbit, this distance achieves the value of the expected error of the GOCE position determination.

In the nearest future, the GOCE mission measurements, i.e. the Earth gravity tensor and the tensors derived from the selected perturbing forces, will be simulated. The properties of these tensors will be determined. These tensors with the simulated GOCE orbit and the approximated variants of this orbit obtained here, along with GPS observations, will be important data in the GOCE satellite orbit improvement process.

\section{REFERENCES}

Anderson J. D., Laing P.A., Lau E. L., et al. Study of the anomalous acceleration of Pioneer 10 and 11. Phys. Rev. D65 082004, 2002.

Bobojć A., Drożyner A. Satellite orbit determination using satellite gravity gradiometry observations in GOCE mission perspective. Advances in Geosciences 1:1-4, 2003.

Bock H., Jäggi A., Svehla D., et al. Precise Orbit Determination for the GOCE Satellite Using GPS. Committee on Space Research 36th COSPAR Scientific Assembly Beijing, China, July 16-23, 2006.

Visser P., Ijssel J., Helleputte T., et al. Rapid and Precise Orbit Determination for the GOCE satellite. 3rd GOCE user workshop, ESA ESRIN, 6-8 November 2006.

Bouman J., Koop R. Error assessment of GOCE SGG data using along track interpolation. Advances in Geosciences 1: 27-32, 2003.

CIRA-72, A. Verlag Akademisches Berlin, 1972.

Ditmar P., Klees R. A Method to Compute the Earth's Gravity Field from SGG/SST data to be Acquired by the GOCE Satellite. Delft University Press, 2002.

Ditmar P., Visser P., Klees R. On the joint inversion of SGG and SST data from the GOCE mission. Advances in Geosciences. 1:87-94, 2003.

Drinkwater, M.R., Floberghagen, R., Haagmans, R., et al. GOCE: ESA's First Earth Explorer Core Mission. Space Science Reviews 00: 1-14, 2003.

Drożyner A. Determination of Orbits with Torun Orbit Processor System. Adv. Space Res., vol. 16, No. 2, 1995. 
ESA. Gravity Field and Steady-State Ocean Circulation Mission, ESA SP-1233(1). Report for mission selection of the four candidate Earth Explorer missions, 1999.

ESA. ESA's Gravity Mission - GOCE Brochure (revised), 2006.

Johannessen J. A., Balmino G., Le Provost C., et al. The European Gravity Field and SteadyState Ocean Circulation Explorer Satellite Mission Its Impact on Geophysics. Surveys in Geophysics; 24(4) : 339-386, July 2003.

Laing P. A. Implementation of J2000.0 reference frame in CHASMP. The Aerospace Corporation's Internal Memorandum \# 91(6703)-1. January 28, 1991.

Lemoine F., Kenyon S., Factor J., et al. The Development of the Joint NASA GSFC and the National Imagery and Mapping Agency (NIMA) Geopotential Model EGM96. Report No.206861, 1998.

Melbourne, W., et al. Project MERIT Standards, Circ. 167, U.S. Naval Observatory, Washington, D.C., 1983.

Mégie G., Readings C. J. The Earth Explorer Missions - Current Status. Earth Observation Quarterly No. 66, 2000.

Wermuth M., Rummel R., Lóránt Földváry. Mission Simulation and Semi-analytical Gravity Field Analysis for GOCE SGG and SST. Observation of the Earth System from Space. Springer Berlin Heidelberg, 2006.

Rebhan, H., Aguirre M., Johannessen J. The Gravity Field and Steady-State Ocean Circulation Explorer Mission - GOCE, ESA Earth Observation Quarterly 66: 6-11, 2000.

Received: 2007-02-16,

Reviewed: 2008-01-15,

Accepted: 2008-01-29. 